\section{Estudo \\ cobebate}

em Gestão

Planejamento
Revista Estudo \& Debate, Lajeado, v. 26, n. 2, 2019. ISSN 1983-036X

DOI: http://dx.doi.org/10.22410/issn.1983-036X.v26i2a2019.1976

\title{
INFLUÊNCIA DO NÍVEL DE ESCOLARIDADE NA PERCEPÇÃO AMBIENTAL DA POPULAÇÃO LOCAL SOBRE O MONUMENTO NATURAL DAS ÁRVORES FOSSILIZADAS DO TOCANTINS (MNAFTO)
}

\author{
Aline Maria Costantin ${ }^{1}$, Daniel de Freitas Nunes ${ }^{2}$, Etiene Fabbrin Pires Oliveira ${ }^{3}$, \\ André Jasper ${ }^{4}$
}

\begin{abstract}
Resumo: O Monumento Natural das Árvores Fossilizadas do Tocantins (MNAFTO), localizado no município de Filadélfia, estado do Tocantins, situado em parte da Amazônia Legal Brasileira, foi objeto de pesquisa para análise da influência do nível de escolaridade no processo de percepção ambiental da populaçáo local sobre esse tipo de unidade de conservação. Dessa forma, foi realizada a análise de correspondência e a estatística descritiva dos resultados coletados por meio da aplicação de entrevistas estruturadas a uma amostra aleatória simples de 382 habitantes do município. Os resultados evidenciaram que os entrevistados $(52,6 \%)$ eram pessoas do sexo feminino com nível médio de escolaridade e idade média de 32,8 anos. O conhecimento dos fósseis ocorreu entre os entrevistados com maior nível de escolaridade. $(42,8 \%)$ e que apresentam os sentimentos de preservação e admiraçáo em relação ao monumento, demonstrando assim a importância do nível de escolaridade no processo de Percepção Ambiental.
\end{abstract}

Palavras-chave: Percepção ambiental. Nível de escolaridade. Análise de correspondência.

1 Professora do Instituto Federal do Tocantins (IFTO). Palmas-TO- Brasil. E-mail: aline@ifto.edu.br, autor correspondente.

2 Professor do Instituto Federal do Tocantins (IFTO). Araguatins-TO-Brasil. E-mail: danielfreitas@uft.edu.br

3 Professora do Programa de Pós-Graduação em Biodiversidade, ecologia e Conservaçấo da Universidade Federal do Tocantins (UFT). Porto Nacional-TO-Brasil. E-mail: etienefabbrin@uft.edu.br

4 Professor do Programa de Pós-Graduação em Ambiente e Desenvolvimento (PPGAD) da Universidade UNIVATES. Lajeado-RS- Brasil. E-mail: ajasper@univates.br 


\title{
INFLUENCE OF THE EDUCATION LEVEL IN THE ENVIRONMENTAL PERCEPTION OF THE LOCAL POPULATION ON THE NATURAL MONUMENT OF THE FOSSILIZED TREES OF TOCANTINS (MANFTO)
}

\begin{abstract}
The Monumento Natural das Árvores Fossilizadas do Tocantins (MNAFTO), located in Filadélfia city, Tocantins State, which is part of the Brazilian Legal Amazon, was the object of research to analyze the influence of educational level on the process of environmental perception of the local population about this type of conservation unit. Thus, the correspondence analysis and the descriptive statistics were performed with the results collected through the application of structured interviews to a simple random sample of 382 inhabitants of the municipality. The results showed that the interviewees (52.6\%) were women with a mean level of schooling and mean age of 32.8 years. The knowledge of the fossils occurred among those interviewed with a higher level of schooling $(42.8 \%)$ and who show the feelings of preservation and admiration in relation to the monument, thus demonstrating the importance of the level of schooling in the process of Environmental Perception.
\end{abstract}

Keywords: environmental perception, level of schooling, correspondence analysis

\section{INTRODUÇÁO}

A Percepção Ambiental ocorre através de um processo que se inicia com a atenção do observador, ou seja, é um processo de observação seletiva, no qual são assimilados alguns elementos em desfavor de outros. Diversos os fatores influenciam neste interesse e podem ser agrupados em duas categorias: os fatores externos (próprios do meio ambiente) e os fatores internos (próprios do organismo humano) (CIRILO, 2007).

Quanto aos fatores internos, é sabido que o indivíduo necessita dos sentidos (visão, olfato, paladar, audição e tato) para ter sensações, formar ideias e compreender o mundo (CIRILO, 2007). Já nos fatores externos, os processos mentais, cognitivos e os simbolismos devem ser associados aos sentidos existentes nos grupos sociais e nos indivíduos. Onde estes últimos tem diferentes valores, culturas e e limites biológicos ou fisiológicos que influenciam na compreensão das relaçóes existentes entre o homem e o meio ambiente (MELAZO, 2005). Dessa forma, acredita-se que os níveis educacionais dos indivíduos podem influenciar na percepçáo ambiental dos mesmos quanto aos fatores externos no âmbito da interpretação e entendimento da relação entre homem e meio ambiente.

Compreendendo a importância da análise desses diferentes níveis de entendimento e interação do homem com o meio ambiente, a Percepçáo Ambiental tem sido tema recorrente em pesquisas recentes que objetivam investigar diferentes níveis de interação e os fatores que os influenciam (TORGLER; GARCIA-VALINAS, 2007; AZMAN et al. 2010; SHAHHOSEINI; MODABBERI; SHAHABI 2016). A importância destas pesquisas reside no entendimento da Percepçáo Ambiental enquanto promotora e provedora de contribuições importantes no âmbito da investigação de saberes, valores, atitudes e opiniōes das pessoas em relação aos diferentes meios em que vivem (SUESS et. al., 2013).

Assim, o presente trabalho objetivou verificar a percepção ambiental a partir dos diferentes níveis de escolaridade da população local que reside no município de Filadélfia, 
estado do Tocantins pertencente a regiáo da Amazônia legal brasileira, onde localiza-se o Monumento Natural das Árvores Fossilizadas do Tocantins (MNAFTO).

\section{MONUMENTO NATURAL DAS ÁRVORES FOSSILIZADAS DO TOCANTINS (MNAFTO) E PERCEPÇÁO AMBIENTAL - ASPECTOS LEGAIS E TEÓRICOS}

A história de criação do MNAFTO remonta ao ano de 1996, em que o Departamento Nacional de Produção Mineral (DNPM) emitiu uma licença de pesquisa para a Mineração Pedra de Fogo Ltda., que, ao invés de pesquisar a região, explorava e comercializava os fósseis de maneira irregular. A Sociedade Brasileira de Paleontologia (SBP) denunciou o ocorrido ao Ministério Público do estado do Tocantins. Após ter ocorrido à denúncia, o governo do Estado criou instrumentos legais com o objetivo de combater a exploraçáo ilegal e proteger os fósseis. Destaca-se a publicação da Medida Provisória n. 370, de 11 de setembro de 2000, cuja matéria se tornou definitiva com o início da vigência da Lei n. 1.1179, de 4 de outubro de 2000, que criou o MNAFTO (MRS/OIKOS, 2005).

O MNAFTO é caracterizado como uma Unidade de Conservação de Proteção Integral da categoria Monumento Natural, com base na legislação federal. Tem como objetivo principal preservar os sítios naturais raros encontrados na regiáo (Lei Federal n. 9.985/2000, art. 12). No MNAFTO, os fósseis são encontrados em afloramentos distribuídos em manchas descontínuas pela área do município de Filadélfia - TO com os fragmentos mais significativos da Floresta Petrificada do Tocantins Setentrional (FPTS) compostos por caules, troncos e folhas fósseis de coníferas e samambaias arborescentes (MRS/OIKOS, 2005).

Devido o MNAFTO ser da categoria monumento natural, é permitida a permanência da população residente na localidade, fomentando assim, a necessidade da compreensão da relação e percepção da população local sobre essas UCs (Brasil, 2000). Dessa forma , para a realização da pesquisa utilizou-se os pressupostos de Tuan (1980), geógrafo humanista precursor da divulgação da temática, para o qual o indivíduo percebe o mundo e o meio em que vive a partir da sua experiência interativa com o mesmo tomando como referência o seu lugar de sujeito no mundo à maneira como age, processo no qual a bagagem cultural de cada ser humano influência de maneira direta na sua atitude.

Beery e Wolf-Watz (2014), ainda descreveram a percepção ambiental como a possível relação cognitiva-emocional do indivíduo com a natureza a partir de experiências que variam conforme a identidade e o tempo gasto com a natureza. Para eles a identidade do indivíduo é formada pelo pensamento sobre si mesmo e a forma como conceitua seu relacionamento com os demais, refletindo assim sobre suas percepçóes, em que os indivíduos agem para preservar as coisas que são emocionalmente ligados e atribuem valor. Já para Mei, Wai e Ahamad (2016), a percepção ambiental é usada como referência para demonstrar as atitudes e sentimentos dos indivíduos em relação ao meio ambiente.

Deste modo, parte-se da compreensão que o estudo da relação da população local com o MNAFTO, insere-se em uma discussão de perspectiva global, visto que a necessidade de entendimento dos recursos naturais como algo finito que demanda uso racional tem sido uma tendência nos estudos gerais que tratam de percepção ambiental (AYENE et al. 2016). 


\section{NOTAS METODOLÓGICAS}

O presente trabalho optou pelo recorte metodológico de um estudo de caso qualitativo instrumental caracterizado pelo enfoque e inferência local e não global dos resultados aferidos e compilados a partir da análise em profundidade de um caso delimitado ancorada em uma extensa coleta de dados que podem fornecer insights para o tema em tela (CRESWEL, 2012).

A coleta de dados partiu de uma delimitaçáo amostral dos prováveis sujeitos da pesquisa. Considerando que o município de Filadélfia - TO (Figura 1), compreende a área do MNAFTO possui, segundo o censo 2010 do IBGE, uma população de 8.505 habitantes, a operacionalização da pesquisa se deu pela extração de uma amostra aleatória simples ${ }^{5}$ de 382 indivíduos (com erro amostral de 5\%), valendo-se do mesmo procedimento amostral utilizado em pesquisas de opiniáo realizada em de 2015 (BARBETTA, 2012). A partir da delimitaçáo da amostra, procedeu-se com a aplicação de questionários estruturados que foram categorizados no software SPSS compreendendo um escopo de variáveis quantitativas e categóricas sobre a percepção dos entrevistados no que diz respeito ao MNAFTO.

Para o tratamento dos dados foi utilizada a análise das variáveis de percepção cruzadas com a escolaridade da amostra e se deu a partir do programa XLSTAT versão 2016 por meio da aplicação da técnica de análise multivariada de dados Análise de Correspondência (AC). De acordo com Hair et al. (2005), a AC possibilita a representação multivariada de interdependência para dados qualitativos, o que se torna inaplicável em outros testes estatísticos. Ainda, segundo Carvalho e Struchiner (1992) esse método se faz eficiente por permitir uma visualização gráfica das relaçóes mais significativas de um grande conjunto de variáveis.

5 O tamanho da amostra foi definido pelas fórmulas $n_{0}=\frac{1}{E_{\mathrm{n}}{ }^{2}}$ e $n=\frac{N \cdot n_{0}}{N+n_{0}}$ onde $N=$ tamanho da população, $E_{0}=$ erro amostral $n_{0=}$ primeira aproximação da amostra e $n=$ tamanho da amostra. 
Figura 1 - Mapa de localização do estado do Tocantins e do MNAFTO.

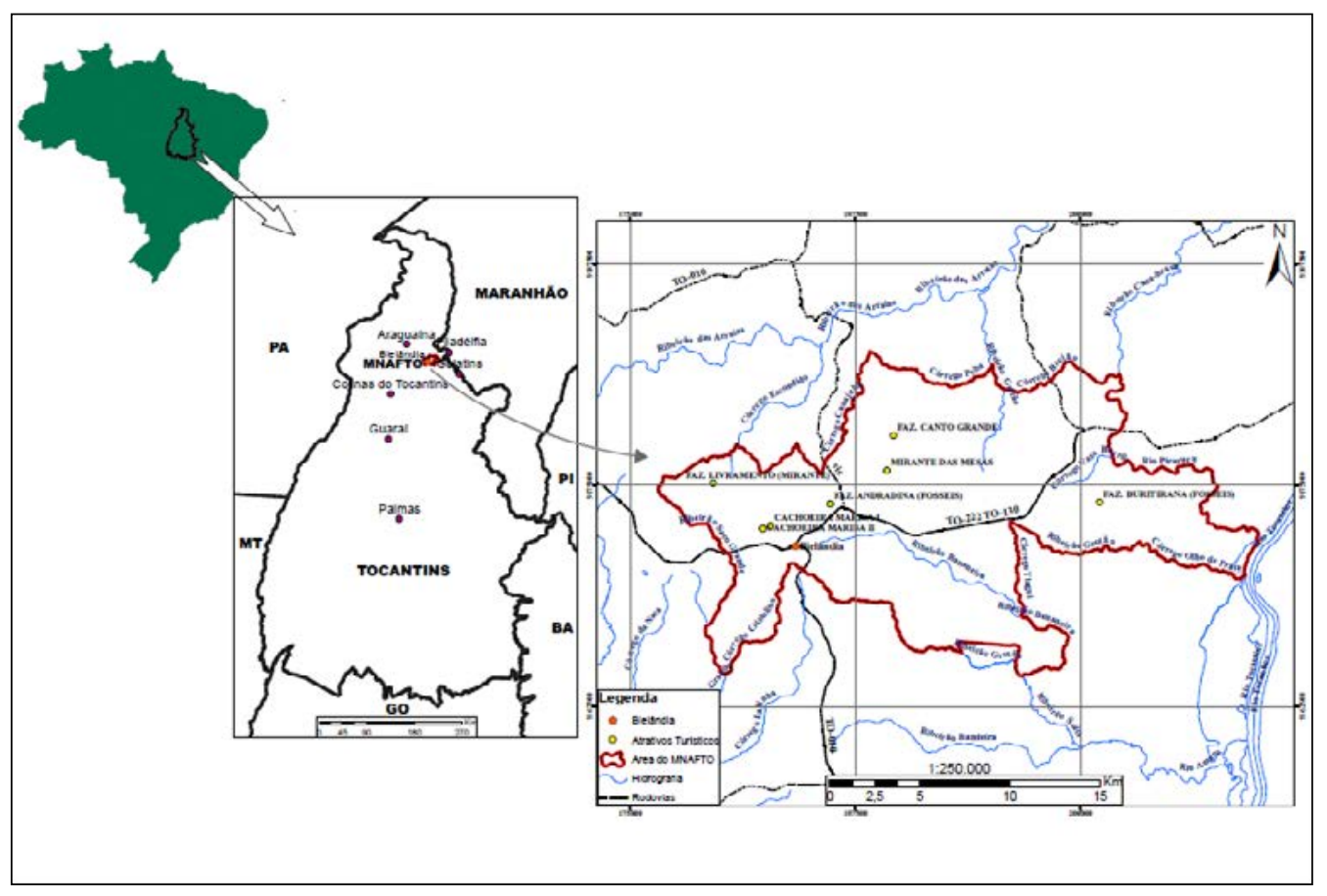

Fonte: NATURATINS (2017)

\section{RESULTADOS E DISCUSSÓES}

Para construir um panorama da Percepção Ambiental da população local em relação ao MNAFTO, foi feita a distribuiçáo amostral a partir das variáveis sexo e escolaridade (Tabela 1 e 2).

Tabela 1- Relaçáo escolaridade e sexo

\begin{tabular}{l|r|r|r|r|r|r}
\hline \multirow{2}{*}{\multicolumn{1}{c|}{ Escolaridade }} & \multicolumn{2}{|c|}{ Sexo feminino } & \multicolumn{1}{c|}{ Sexo masculino } & \multicolumn{2}{c}{ Total } \\
\cline { 2 - 7 } & \multicolumn{1}{c|}{$\mathbf{n}^{\circ}$} & \multicolumn{1}{c}{$\boldsymbol{\%}$} & \multicolumn{1}{c}{$\mathbf{n}^{\circ}$} & \multicolumn{1}{c}{$\boldsymbol{\%}$} & \multicolumn{1}{c}{$\mathbf{n}^{\circ}$} & \multicolumn{1}{c}{$\%$} \\
\hline não lê, nem escreve & 7 & 1,83 & 9 & 2,36 & 16 & 4,19 \\
nível fundamental & 47 & 12,30 & 63 & 16,50 & 110 & 28,80 \\
nível médio & 95 & 24,86 & 97 & 25,40 & 192 & 50,26 \\
nível superior & 52 & $\mathbf{1 3 , 6 1}$ & 12 & 3,14 & 64 & 16,75 \\
\hline Total & 201 & $\mathbf{5 2 , 6 0}$ & 181 & 47,40 & 382 & 100 \\
\hline
\end{tabular}

Fonte: dados da pesquisa.

A maioria dos entrevistados (52,6\%) são do sexo feminino (Tabela 1). Em ambos os sexos, a escolaridade de nível médio é a preponderante (50,26\%). Houve uma diferença 
significativa entre os sexos quando se trata do nível superior de escolaridade, sendo que $13,61 \%$ das mulheres possuem nível superior e somente 3,14\% dos homens. A idade média dos entrevistados é de 32,8 anos, mínima de 12 anos e a máxima de 91 anos com desvio padrão de 16,9. De acordo com o censo do Instituto Brasileiro de Geografia e Estatística - IBGE (2010), a população do município de Filadélfia é formada por 4.470 homens $(52,56 \%)$ e 4.035 mulheres (47,44\%), os percentuais amostrados neste estudo refletem de maneira inversa a constituição da população. Essa discussão é importante na medida em que, com relação á escolaridade nível superior é marcante. $\mathrm{Na}$ amostragem utilizada neste estudo, o nível superior é bem maior entre as mulheres. A Tabela 2 consistiu na verificação dos níveis de escolaridades compilados e a percepção do material fossilífero do MNAFTO. Essa verificação permitiu um maior entendimento do tema dentro dos estratos de indivíduos com ensino médio ( $71,3 \%$ declararam saber da existência de fósseis no monumento) e ensino superior $(73,4 \%)$.

Tabela 2- Tabela de frequência "Conhecimento dos fósseis em relação a escolaridade"

\begin{tabular}{|c|c|c|c|c|c|c|c|c|c|c|c|c|}
\hline \multirow{3}{*}{$\begin{array}{l}\text { Você sabe o que } \\
\text { sáo fósseis? }\end{array}$} & \multicolumn{12}{|c|}{ Escolaridade } \\
\hline & \multicolumn{3}{|c|}{ Náo lê, nem escreve } & \multicolumn{3}{|c|}{ Nível fundamental } & \multicolumn{3}{|c|}{ Nível médio } & \multicolumn{3}{|c|}{ Nível superior } \\
\hline & $\mathbf{N}^{*}$ & $\%$ g. ${ }^{* *}$ & \%c.*** & $\mathbf{N}^{*}$ & $\%$ g.** & $\% c^{* * *}$ & $\mathbf{N}^{*}$ & $\%$ g.** & $\%$ c. $^{* * *}$ & $\mathbf{N}^{*}$ & $\%$ g. ${ }^{* *}$ & $\%$ c. $^{* * *}$ \\
\hline Sim & 8 & 2,10 & 50 & 58 & 15,18 & 52,72 & 137 & 35,90 & 71,36 & 47 & 12,30 & 73,44 \\
\hline Não & 5 & 1,30 & 31,25 & 42 & 10,99 & 38,18 & 23 & 6,02 & 11,98 & 6 & 1,57 & 9,37 \\
\hline $\begin{array}{l}\text { tenho alguma } \\
\text { noção }\end{array}$ & 3 & 0,78 & 18,75 & 5 & 1,30 & 4,55 & 21 & 5,50 & 10,93 & 9 & 2,36 & 14,06 \\
\hline $\begin{array}{l}\text { já ouvi falar, } \\
\text { mas náo sei } \\
\text { realmente o } \\
\text { que é }\end{array}$ & - & - & - & 5 & 1,30 & 4,545 & 11 & 2,88 & 5,73 & 2 & 0,52 & 3,13 \\
\hline Total & 16 & 4,18 & 100 & 110 & 28,77 & 100 & 192 & 50,30 & 100 & 64 & 16,75 & 100 \\
\hline
\end{tabular}

*Número de entrevistados

** \%g.= percentual sobre o total geral

*** \%c. = percentual sobre a categoria

Fonte: dados da pesquisa.

A interação multivariada dessas variáveis a partir da análise de correspondência indicou uma associação clara entre os níveis de escolaridades verificados e a percepção dos fósseis no MNAFTO (Gráfico 1). 
Gráfico 1- Gráfico simétrico gerado pela análise de correspondência "Conhecimento dos fósseis em relação a escolaridade"

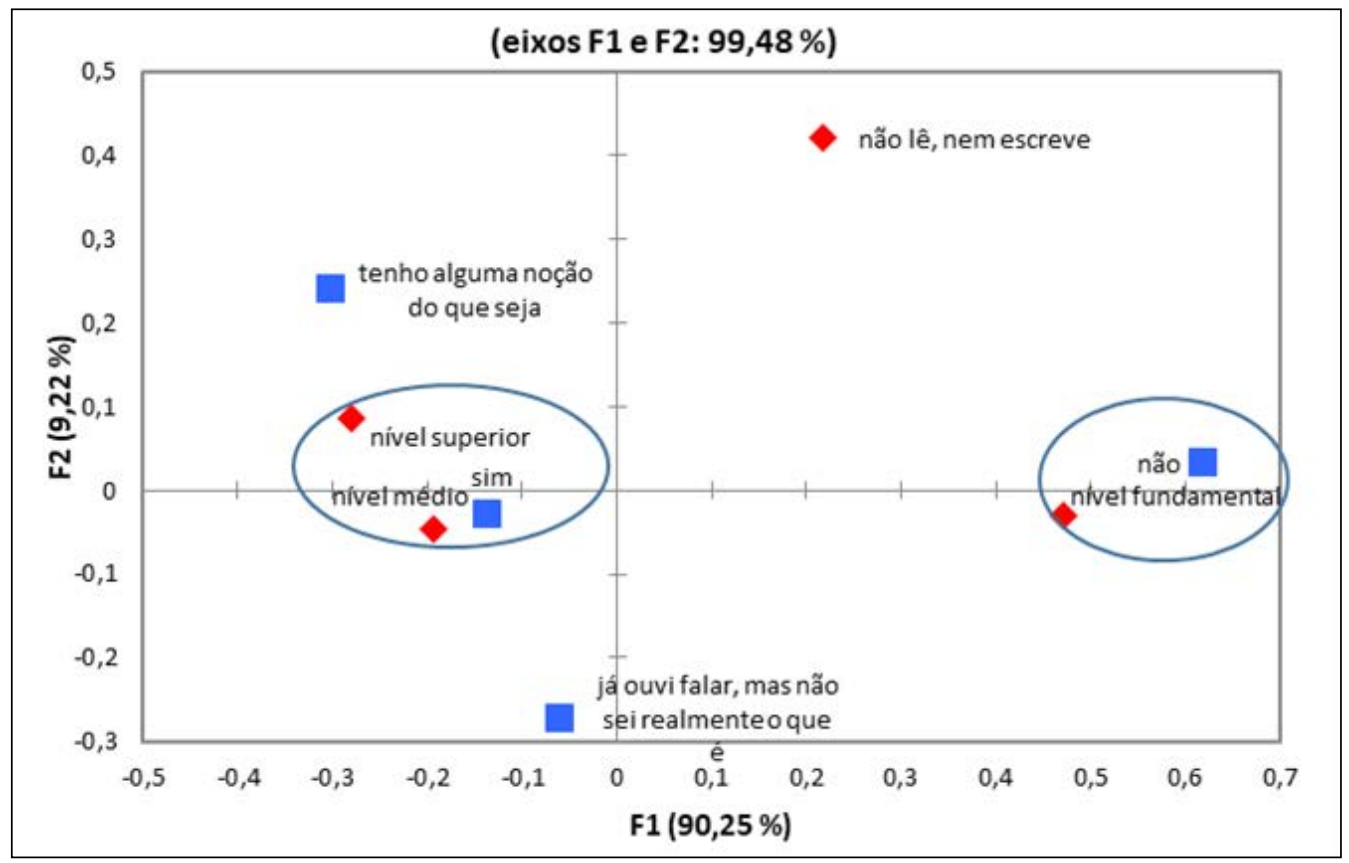

Fonte: dados da pesquisa.

A associação entre os níveis de escolaridade mais elevados e a percepção positiva de caracteres que compóem o meio ambiente é relatada em diversos estudos (e.g. BLOMQUIST; WHITEHEAD, 1998; TORGLER; GARCÍA-VALIÑAS, 2007; MEYER, 2015) não sendo diferente na amostra trabalhada (Gráfico 1). Quanto à percepção relacionada à UC's, os dados também corroboram os resultados de Magalhães et al (2010) que avaliaram a participação da sociedade civil na gestáo de UCs no estado do Mato Grosso do Sul, Brasil; os autores verificaram proporção direta entre escolaridade e conhecimento das questóes ambientais. Sucintamente, estas pesquisas têm demonstrado que a escolaridade pode afetar a responsabilidade pessoal, a ética e a consciência social. Nesse sentido, a identificação destas correspondências pode auxiliar no desenho e aplicação de atividades de conscientização direcionadas para grupos prioritários (indivíduos com ensino fundamental) aproveitando o capital humano local (indivíduos com nível médio e superior) o que pode vir a favorecer a percepção ambiental (MEYER, 2015).

Ao realizar a análise entre o conhecimento sobre o estado de preservaçáo dos fósseis e a escolaridade (Tabela 3), observa-se que os dados quantitativos gerais demonstram que os entrevistados em todos os níveis de escolaridade percebem que os fósseis são preservados, sendo eles bem preservados, preservados ou pouco preservados. Entretanto, entre os entrevistados que possuem nível médio de escolaridade, foi evidenciado o maior percentual geral $(9,35 \%)$ de indivíduos que acham que os fósseis não são preservados. 
Tabela 3- Tabela de frequência "Estado de preservação dos fósseis em relação a escolaridade"

\begin{tabular}{|c|c|c|c|c|c|c|c|c|c|c|c|c|}
\hline \multirow{3}{*}{$\begin{array}{l}\text { Você acha } \\
\text { que os fósseis } \\
\text { encontrados } \\
\text { na regiáo são } \\
\text { preservados? }\end{array}$} & \multicolumn{12}{|c|}{ Escolaridade } \\
\hline & \multicolumn{3}{|c|}{ Náo lê, nem escreve } & \multicolumn{3}{|c|}{ Nível fundamental } & \multicolumn{3}{|c|}{ Nível médio } & \multicolumn{3}{|c|}{ Nível superior } \\
\hline & $\mathbf{N}^{* *}$ & $\%$. $^{* * *}$ & $\% c . * * * *$ & $\mathbf{N}^{* *}$ & $\%$. $^{* * *}$ & $\%$ c.**** $^{*}$ & $\mathbf{N}^{* *}$ & \%g.*** & $\%$ c. $^{* * * *}$ & $\mathbf{N}^{* *}$ & \%g.*** & $\%$ c. $^{* * * *}$ \\
\hline Sim & 7 & 2,10 & 63,64 & 45 & 13,55 & 56,25 & 90 & 27,10 & 49,45 & 35 & 10,54 & 59,32 \\
\hline $\begin{array}{l}\text { sáo bem } \\
\text { preservados }\end{array}$ & 1 & 0,30 & 9,09 & 9 & 2,71 & 11,25 & 29 & 8,80 & 15,93 & 4 & 1,20 & 6,78 \\
\hline $\begin{array}{l}\text { são pouco } \\
\text { preservados }\end{array}$ & 2 & 0,60 & 18,18 & 14 & 4,21 & 17,5 & 32 & 9,63 & 17,58 & 13 & 3,91 & 22,03 \\
\hline $\begin{array}{l}\text { náo são } \\
\text { preservados }\end{array}$ & 1 & 0,30 & 9,09 & 12 & 3,6 & 15 & 31 & 9,35 & 17,04 & 7 & 2,10 & 11,87 \\
\hline Total & 11 & 3,30 & 100 & 80 & 24,07 & 100 & 182 & 54,88 & 100 & 59 & 17,75 & 100 \\
\hline
\end{tabular}

* 50 indivíduos não responderam esta questão.

**Número de entrevistados

*** \%g.= percentual sobre o total geral

$* * * * \% c .=$ percentual sobre a categoria

Fonte: dados da pesquisa.

Em relação à análise de correspondência entre o nível de escolaridade e o sentimento com relação à preservação dos fósseis (Gráfico 2), ocorreram correspondências entre os indivíduos de nível fundamental e o sentimento de preservação e entre os indivíduos de nível médio e o sentimento de que não são bem preservados. Dessa forma, foi evidente que os entrevistados com maior nível de escolaridade (médio) são mais exigentes em relação à conservação da natureza, tal como relatado por Meyer (2015). Além disso, os autores acreditam que esta percepção de preservação esteja relacionada ao conceito de que as UCs devam ser áreas protegidas e intocadas, caso este que não ocorre no MNAFTO devido justamente ao fato de ser uma UC do tipo monumento natural. 
Gráfico 2- Gráfico simétrico gerado pela análise de correspondência "Estado de conservação dos fósseis em relação a escolaridade"

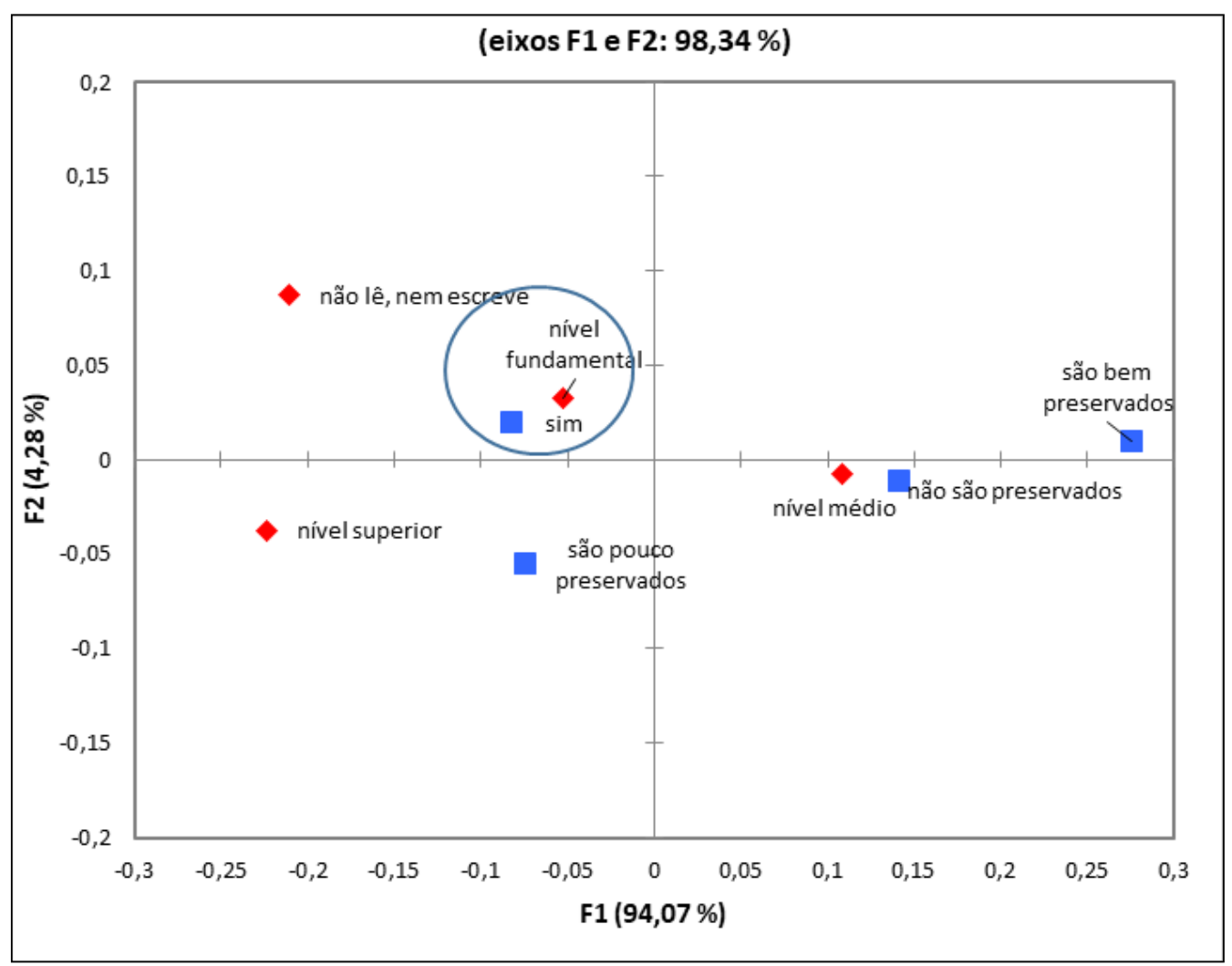

Fonte: dados da pesquisa.

Este tipo de percepção de UCs como áreas protegidas e intocadas já foi observada por Bresolin, Zakrzevski e Marinho (2010), quando realizaram um estudo sobre percepção, comunicação e Educação Ambiental em unidades de conservação no parque estadual de Espigão Alto - Barracão/RS - Brasil. Mesmo assim, grande parte da população entrevistada considerou o MNAFTO como preservado, corroborando com Kauffmann (2014, p. 21), quando se refere que apesar dos diferentes estudos em relação aos fitofósseis que afloram no MNAFTO e seu entorno, "a abundância e o excelente grau de preservação têm se mostrado de incontestável importância patrimonial em âmbito nacional e mundial”. Entre as possíveis explicações sobre esse entendimento de preservação do Patrimônio, Azman et al. (2011) discutem que a percepção e o entendimento do Patrimônio Geológico vão além do nível educacional da comunidade local. Os autores indicam que as açóes sistemáticas de conscientização são fundamentais para melhorar essa compreensão, como as desenvolvidas na região do MANFTO.

A quarta tabela foi referente à percepção da influência que a existência do MNAFTO sobre a vida da população local (Tabela 4), na qual foi evidenciado para $70 \%$ dos entrevistados, o monumento não exerce nenhuma influência em suas vidas, sendo estes 
resultados mais evidentes nas respostas dos indivíduos com ensino médio. Apesar disso, $16 \%$ dos entrevistados perceberam o monumento como "fonte de renda" (Gráfico 3).

Tabela 4- Tabela de frequência sobre o tema "Influência na vida da populaçáo causada pelo MNAFTO em relação a escolaridade"

\begin{tabular}{|c|c|c|c|c|c|c|c|c|c|c|c|c|}
\hline \multirow{3}{*}{$\begin{array}{l}\text { O MNAFTO influencia } \\
\text { na sua vida de alguma } \\
\text { maneira? }\end{array}$} & \multicolumn{12}{|c|}{ Escolaridade } \\
\hline & \multicolumn{3}{|c|}{ Náo lê, nem escreve } & \multicolumn{3}{|c|}{ Nível fundamental } & \multicolumn{3}{|c|}{ Nível médio } & \multicolumn{3}{|c|}{ Nível superior } \\
\hline & $\mathbf{N}^{* *}$ & \%g. ${ }^{* * *}$ & \%c. ${ }^{* * * *}$ & $\mathbf{N}^{* *}$ & \%g.*** & $\% \mathrm{c}^{* * * *}$ & $\mathbf{N}^{* *}$ & \%g. ${ }^{* * *}$ & \%c.**** & $\mathbf{N}^{* *}$ & \%g.*** & \%c.**** \\
\hline nenhuma & 11 & 2,96 & 73,33 & 75 & 20,16 & 71,43 & 129 & 34,67 & 68,25 & 47 & 12,63 & 74,60 \\
\hline fonte de renda & 1 & 0,27 & 6,67 & 20 & 5,37 & 19,04 & 32 & 8,60 & 16,94 & 7 & 1,89 & 11,11 \\
\hline atrapalha & 1 & 0,27 & 6,67 & 4 & 1,07 & 3,80 & 5 & 1,34 & 2,64 & - & - & - \\
\hline outra & 2 & 0,54 & 13,33 & 6 & 1,62 & 5,73 & 23 & 6,19 & 12,17 & 9 & 2,42 & 14,29 \\
\hline Total & 15 & 4,04 & 100 & 105 & 28,22 & 100 & 189 & 50,80 & 100 & 63 & 16,94 & 100 \\
\hline
\end{tabular}

*10 indivíduos não responderam esta questão.

** Número de entrevistados

*** \%g.= percentual sobre o total geral

**** \%c. = percentual sobre a categoria

Fonte: dados da pesquisa.

Gráfico 3- Gráfico simétrico gerado pela análise de correspondência com o tema "Influência na vida causada pelo MNAFTO em relaçáo a escolaridade"

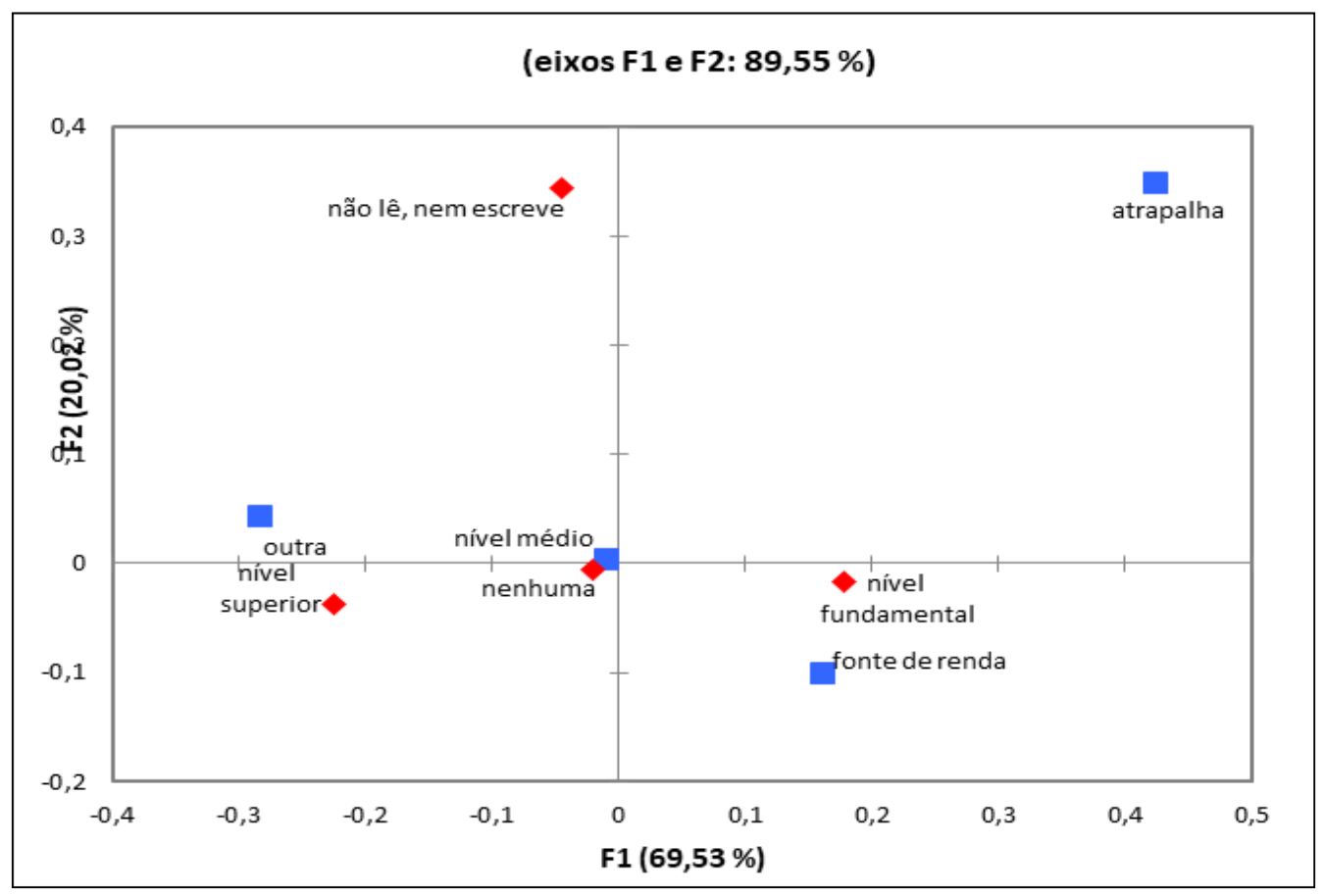

Fonte: dados da pesquisa. 
Apesar de $70 \%$ dos entrevistados não terem percebido influência em suas vidas causadas pelo MNAFTO uma parcela da população, representada pelos entrevistados evidenciou o monumento como fonte de renda (16,13\%). Este fato pode ter sido causado pelas atividades proporcionadas através da implantaçăo do MNAFTO. Pois, os visitantes acompanhados de guias locais treinados podem representar incrementos na renda da população local, além de aumentarem a renda da comunidade através do consumo de alimentos (CASTRO, NORONHA E MEDEIROS, 2016). Ainda para Azman et al. (2011) essa percepção sobre benefícios econômicos gerados pelo geoturismo, dependem fundamentalmente de ações de Educação Ambiental voltadas para populaçáo local e direcionadas para o patrimônio geológico.

A quinta tabela consistiu na verificação da percepção afetiva em relação ao monumento (Tabela 5), foi evidenciado o sentimento de "preservação" como maior referência para os entrevistados, principalmente da categoria com nível médio de escolaridade (Gráfico 4).

Tabela 5- Tabela de frequência sobre o tema "Sentimento sobre o MNAFTO em relação a escolaridade"

\begin{tabular}{|c|c|c|c|c|c|c|c|c|c|c|c|c|}
\hline \multirow{3}{*}{$\begin{array}{l}\text { Qual seu sentimento em } \\
\text { relaçáo ao MNAFTO? }\end{array}$} & \multicolumn{12}{|c|}{ Escolaridade } \\
\hline & \multicolumn{3}{|c|}{ Náo lê, nem escreve } & \multicolumn{3}{|c|}{ Nível fundamental } & \multicolumn{3}{|c|}{ Nível médio } & \multicolumn{3}{|c|}{ Nível superior } \\
\hline & $\mathbf{N}^{*}$ & $\% g . * *$ & $\%$ c. $^{* * *}$ & $\mathbf{N}^{*}$ & $\% g . * *$ & $\%$ c. $^{* * *}$ & $\mathbf{N}^{*}$ & $\% g . * *$ & $\%$ c. $^{* * *}$ & $\mathbf{N}^{*}$ & $\%$ g. ${ }^{* *}$ & $\%$ c. $^{* * *}$ \\
\hline nenhum & 9 & 2,37 & 52,94 & 39 & 10,21 & 36,44 & 50 & 13,08 & 25,90 & 12 & 3,14 & 18,46 \\
\hline preservação & 5 & 1,30 & 29,41 & 35 & 9,17 & 32,71 & 85 & 22,25 & 44,05 & 32 & 8,38 & 49,23 \\
\hline admiração & 2 & 0,52 & 11,76 & 28 & 7,33 & 26,17 & 52 & 13,61 & 26,95 & 20 & 5,25 & 30,76 \\
\hline ódio-raiva & 1 & 0,26 & 5,89 & 5 & 1,30 & 4,68 & 6 & 1,57 & 3,10 & 1 & 0,26 & 1,55 \\
\hline Total & 17 & 4,45 & 100 & 107 & 28,01 & 100 & 193 & 50,51 & 100 & 65 & 17,03 & 100 \\
\hline
\end{tabular}

* Número de entrevistados

** \%g.= percentual sobre o total geral

*** \%c. = percentual sobre a categoria

Fonte: dados da pesquisa. 
Gráfico 4- Gráfico simétrico da análise de correspondência com o tema "Sentimento sobre o MNAFTO em relação a escolaridade”

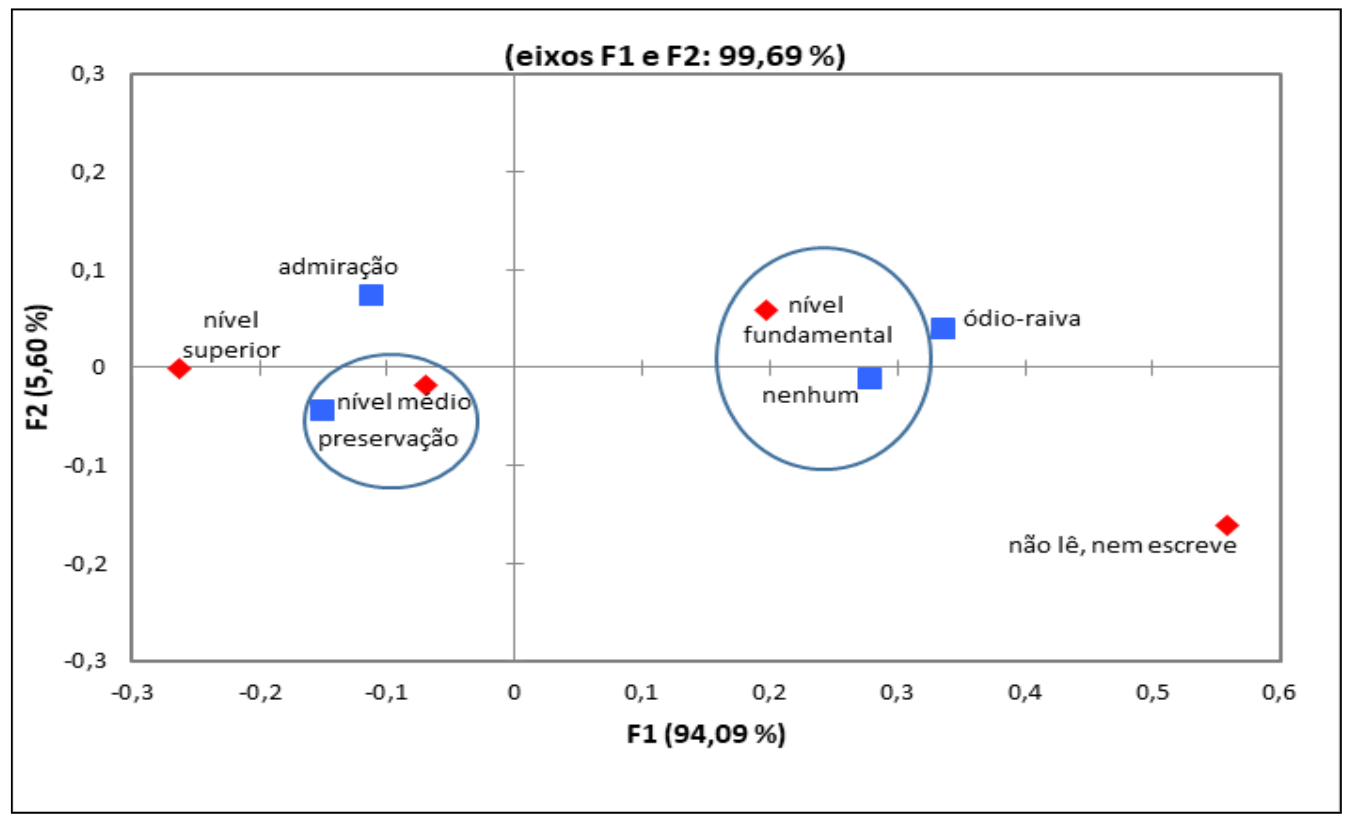

Fonte: dados da pesquisa.

Nas análises da percepção da população local sobre o MNAFTO os sentimentos apresentados de maneira preponderante pelos entrevistados foram os de preservação e admiração, que tendem a ser maiores nos níveis educacionais mais elevados. Segundo Bispo e Oliveira (2007) os sensos de preservação e admiração são impregnados de açóes e sensações apreendidas e vivenciadas no lugar que permitem o entendimento das representaçóes ambientais. No qual indicam caminhos e açóes educacionais a serem seguidas como os resultados encontrados nas análises das percepções em relação ao monumento.

Em relação aos sentimentos de preservação e admiração sobre o MNAFTO, de acordo com Azman et al. (2010) e Bento e Rodrigues (2013) eles podem apresentar um viés educativo para a população local, as noçôes de respeito e conservação ambiental possibilitam a valorização da geodiversidade. Além disso, para Mei, Wai e Ahamad (2016) o comportamento ambiental benéfico é ativado pela consciência, preocupaçáo e responsabilidade do indivíduo como meio ambiente. Nesse sentido, os resultados encontrados corroboram com o pensamento dos autores, pois os sentimentos positivos de preservação e admiração aumentaram quando comparadas as categorias específicas nível médio com a de nível superior de escolaridade.

\section{CONSIDERAÇÓES FINAIS}

A análise da Percepção Ambiental da população local sobre o Monumento Natural das Árvores Fossilizadas do Tocantins (MNAFTO) demonstrou que: 
a) o perfil da população local era formado em sua maioria por pessoas do sexo feminino e com nível de escolaridade médio;

b) entrevistados com os níveis mais elevados de escolaridade afirmaram conhecer os fósseis encontrados na região, refletindo a importância da educação no processo de percepção ambiental;

c) a maioria dos entrevistados percebeu que os fósseis são preservados;

d) mais da metade dos entrevistados, não percebeu a influência do MNAFTO em suas vidas;

e) um pequeno grupo possuidor do nível fundamental de escolaridade percebeu o monumento como fonte de renda;

f) em todos os níveis de escolaridade foram presentes os sentimentos de preservação e admiração em relação ao MNAFTO.

\section{REFERÊNCIAS}

AYENE, A. O. et al. The local experts' perception of environmental change and its impacts on surface water in Southwestern Nigeria. Environmental Development, n. 17, 33-47, 2016.

AZMAN, N. et al. Public Education in Heritage Conservation for Geopark Community. Procedia - Social and Behavioral Sciences, v. 7, p. 504-5011, 2010.

. The Langkawi Global Geopark: local community's perspectives on public education. International Journal of Heritage Studies, v. 17, n. 3, p. 261-279, 2011.

BARBETTA, P. A. Estatística aplicada às ciências sociais. 8. ed. Florianópolis: UFSC, 2012.

BEERY, T. H.; WOLF-WATZ, D. Nature to place: Rethinking the environmental connectedness perspective. Journal of Environmental Psychology, n. 40, p. 198-205, 2014.

BENTO, L. C. M.; RODRIGUES, S. C. Geoturismo em Unidades de Conservação: uma nova tendência ou uma necessidade real? Estado da arte. Revista do Departamento de Geografia USP, v. 25, p. 77-97, 2013.

BISPO, M. O.; OLIVEIRA, S. F. Lugar e cotidiano: categorias para compreensão de representações em meio ambiente e educação ambiental. Revista Brasileira de Educação Ambiental, Brasília, n. 2, p. 71-78, 2007.

BLOMQUIST, G. C.; WHITEHEAD, J. C. Resource quality information and validity of willingness to pay in contingent valuation. Resource and Energy Economics, n. 20, 179-196, 1998. 
BRASIL. Sistema Nacional de Unidades de Conservação da Natureza. Lei no 9.985, de 18 de julho de 2000; Decreto no 4.340 de 22 de agosto de 2002. 2. ed. Brasília: SNUC, 2000.

BRESOLIN, A. J.; ZAKRZEVSKI, S. B. B.; MARINHO, J. R. Percepção, Comunicação e Educação Ambiental em Unidades de Conservação: um estudo no Parque Estadual de Espigão Alto - Barracão/RS - Brasil. Perspectiva, Erechim. v. 34, n.128, p. 103-114, 2010 .

CARVALHO, M. S.; STRUNCHINER, C. J. Análise de Correspondência: uma aplicação do método à avaliação de serviços de vacinação. Caderno de Saúde Pública, Rio de Janeiro, Vol. 8, n. 3, pp. 287-301, jul/set. 1992. Disponível em: <http://www.scielo.br/ pdf/csp/v8n3/v8n3a08>. Acesso em: 1 out. 2016.

CASTRO, L. L. C.; NORONHA, G. S.; MEDEIROS, M. A. A. Ecoturismo como alternativa de Desenvolvimento Socioeconômico na Ilha do Cajual, Alcântara (MA). Revista Brasileira de Ecoturismo, São Paulo, v. 9, n. 3, p. 418-432, 2016.

CIRILO, C. L. B. A Comunidade do Cabocó e a Percepção Ambiental das Crianças e Adolescentes para a Gestáo do Rio Capibaribe. Dissertação (Mestrado). Programa de Pós-graduação em Gestão e Políticas Ambientais, Universidade Federal de Pernambuco, Recife, 2007.

CRESWELL, J. W. Educational research: Planning, conducting, and evaluating quantitative and qualitative research. 4. ed. Boston: Pearson, 2012.

HAIR, J. F. et al. Análise multivariada de dados. 5. ed. São Paulo: Artmed, 2005.

IBGE. Instituto Brasileiro de Geografia e Estatística. Censo 2010. Disponível em: <http://www.ibge.gov.br/cidadesat/topwindow.htm?1>. Acesso em: 21 set. 2016.

KAUFFMANN, M. Mapeamento Geológico e Levantamento Paleontológico do Monumento Natural das Árvores Fossilizadas, Bacia do Parnaíba, Estado do Tocantins. Tese (Doutorado). Programa de Pós-Graduação Instituto de GeociênciasUNICAMP, Campinas, 2014.

MAGALHÂES, H.; BONONI, V. L. R.; MERCANTE, M. A. Participação da sociedade civil na gestão de unidades de conservação e seus efeitos na melhoria da qualidade ambiental da regiáo Sudeste do Estado do Mato Grosso do Sul. Acta Scientiarum Human and Social Sciences, Maringá, v. 32, n. 2, p. 183-192, 2010.

MEI, N. S.; WAI, C. W.; AHAMAD, R. Environmental Awareness and Behaviour Index for Malaysia. Procedia - Social and Behavioral Sciences, v. 222, p. 668-675, 2016. 
MELAZO, G.C. Percepção Ambiental e Educação Ambiental: uma reflexão sobre as relaçôes interpessoais e ambientais no espaço urbano. Olhares \& Trilhas, Ano VI, n. 6, p. $45-51,2005$.

MEYER, A. Does education increase pro-environmental behavior? Evidence from Europe. Ecological Economics, n. 116, p. 108-121, 2015.

MRS/OIKOS. Plano de Manejo do Monumento Natural das Árvores Fossilzadas do Tocantins. Planos de manejo e de uso público no Monumento Natural das Árvores Fossilizadas do Tocantins e diagnóstico biofísico e sócio-econômico. 2005. Brasília: MRS, 2005.

NATURATINS. Instituto Natureza do Tocantins. Mapa de Localizaçáo do Monumento Natural das Árvores Fossilizadas. Palmas-TO, 2017.

SHAHHOSEINI, H.; MODABBERI, S.; SHAHABI, M. Study of Factors Influencing the Attitude of Local People Toward Geotourism Development in Qeshm National Geopark, Iran. Geoheritage, 2016. DOI: 10.1007/s12371-015-0171-y

SUESS, R. C.; BEZERRA, R. G.; CARVALHO SOBRINHO, H. Percepção Ambiental de Diferentes Atores Sociais sobre o Lago do Abreu em Formosa-GO. HOLOS, Ano 29, v. 6, p. 241-261, 2013.

TOCANTINS. Assembleia Legislativa do Tocantins. Medida Provisória no 370, de 11 de setembro de 2000. Disponível em: <http://www.al.to.gov.br/ legislacaoEstadual?pagPaginaAtual=88>. Acesso em: 1 set. 2016.

. Lei $\mathrm{n}^{\circ} \mathbf{1 . 1 7 9}$, de 4 de outubro de 2000. Monumento natural das árvores fossilizadas do Tocantins. Disponível em: <http://gesto.to.gov.br/uc/44/>. Acesso em: 1 set. 2016.

TORGLER, B.; GARCÍA-VALIÑAS, M.A. The determinants of individuals' attitudes towards preventing environmental damage. Ecological Economics, n. 63, p. 536-552, 2007.

TUAN, YU-FU. Topofilia - Um Estudo da Percepção, Atitudes e Valores do Meio Ambiente. Tradução Lívia de Oliveira, Rio Claro: UNESP, 1980. 